(1)

CrossMark

\title{
Looking at the COPD spectrum through "PRISm"
}

\author{
Amin Adibi and Mohsen Sadatsafavi (D)
}

Affiliation: Respiratory Evaluation Sciences Program, Collaboration for Outcomes Research and Evaluation, Faculty of Pharmaceutical Sciences, University of British Columbia, Vancouver, BC, Canada.

Correspondence: Mohsen Sadatsafavi, Faculty of Pharmaceutical Sciences, University of British Columbia, Vancouver, BC, Canada. E-mail: msafavidmail.ubc.ca

@ERSpublications

Preserved ratio impaired spirometry (PRISm) is a prevalent, neglected condition whose prognosis is close to that of COPD http://bit.ly/2Oydvq1

Cite this article as: Adibi A, Sadatsafavi M. Looking at the COPD spectrum through "PRISm". Eur Respir J 2020; 55: 1902217 [https://doi.org/10.1183/13993003.02217-2019].

COPD is a major burden globally. According to the Global Burden of Disease study, COPD caused 3.2 million deaths in 2015, accounting for 5\% of all deaths worldwide, making it the third leading cause of death in the world [1]. The Global Initiative for Chronic Obstructive Lung Disease (GOLD) defines spirometrically confirmed COPD based on a forced expiratory volume during the first second $\left(\mathrm{FEV}_{1}\right)$ to a forced vital capacity (FVC) ratio smaller than 0.7 [2]. The severity of airflow obstruction is further defined through GOLD severity grades based on the ratio of $\mathrm{FEV}_{1}$ to its predicted value, with GOLD 1, 2, 3 and 4 defined around cut-off points of $80 \%, 50 \%$, and $30 \%$ [2].

While diagnostic and disease management decisions (e.g. therapeutic choices) demand definitions that create distinct categories, the physiological processes underlying COPD act on a continuous scale [3]. For example, it is recognised that patients fall on a continuous spectrum on the three major aspects of COPD: rate of lung function decline [4], frequency of acute COPD exacerbations [5] and symptom burden [6], with little correlation between the three. Categorising such a continuous process inevitably results in COPD phenotypes that are numerous, loosely defined, and not always mutually exclusive $[7,8]$.

In this context, the two-pronged definition of COPD and its severity grades creates categories that cannot be directly classified as either normal lung function or COPD. One such category comprises patients who have reduced $\mathrm{FEV}_{1}\left(\mathrm{FEV}_{1}<80 \%\right.$ pred) and reduced $\mathrm{FVC}$, in such a way that $\mathrm{FEV}_{1} / \mathrm{FVC}$ $\geqslant 0.7$. Patients in this population, known as preserved ratio impaired spirometry (PRISm), are classified as having reduced lung function but do not meet the spirometry definition of COPD. For that, such patients have largely been excluded from major therapeutic trials [9]. As such, characteristics of these populations, and their prognosis and appropriate treatments, remain poorly defined. Given that PRISm resembles COPD in term of reduction in $\mathrm{FEV}_{1}$, but not in terms of $\mathrm{FEV}_{1} / \mathrm{FVC}$ ratio, a natural question is whether the prognosis in such a "intermediate" group is closer to that of COPD or that of the general non-COPD population.

The study by Wijnant et al. [10], published in this issue of the European Respiratory Journal, tackles such a question head on, and provides important insights on PRISm prevalence, trajectory, and prognosis at the population level. The authors have used the well-known Rotterdam study, an ongoing population-based prospective cohort [11], to investigate the prevalence and prognosis of PRISm in older adults over a 10 -year period. Unlike most of the similar studies published previously, this was a population-based study 
that also included never-smokers. Each individual was categorised as COPD, PRISm, or control (normal spirometry) in each of the two study visits (which were on average 4.6 years apart). Incident COPD and incident PRISm were defined as satisfying the corresponding definitions in the second visit while being classified as a control in the first visit. Similarly, persistent COPD and persistent PRISm were defined as satisfying the corresponding definition in both visits.

Based on a sample of 5487 subjects, the authors report a prevalence of $7.1 \%$ for PRISm in adults $\geqslant 45$ years of age $\left(5.1 \%\right.$ when $\mathrm{FEV}_{1} / \mathrm{FVC}$ cut-off was based on the lower limit of normal criterion). Compared with controls and COPD groups, PRISm was the most unstable in terms of transitioning to other groups: more than $60 \%$ of both control and COPD groups in the first visit remained as such in the second visit; this value was only $23 \%$ for PRISm. The instability of PRISm is not surprising, given that its definition puts it in between COPD and control groups.

There is also evidence for a potential role of obesity on transition into and out of PRISm. Subjects with persistent PRISm generally had higher body mass index at baseline, while subjects with incident PRISm had higher likelihood of gaining weight between the visits. This can be attributable to obesity resulting in a restrictive spirometry pattern and reduction in $\mathrm{FVC}$, while preserving the $\mathrm{FEV}_{1} / \mathrm{FVC}$ ratio.

The most important findings of this study, however, are about the outcomes. PRISm was associated with significant risk of mortality, especially cardiovascular mortality. WIJNANT et al. [10] reported a mortality rate of $18.7 \%$ for PRISm patients, which is considerably higher than the $10.3 \%$ rate in the control group and only slightly lower than the $20.8 \%$ in the COPD group. Compared to subjects with persistent normal spirometry, mortality rate ratio was highest in subjects with incident PRISm (3.8), followed by persistent PRISm (3.2), persistent COPD (1.8) and incident COPD (1.4). PRISm patients had a particularly higher prevalence of cardiovascular comorbidity, and subsequently a higher rate of cardiovascular mortality (hazard ratio (HR) 2.8 compared to controls), larger than that of COPD patients (HR 2.1 compared to controls), though one must be cautious in interpreting these results given the relatively small sample size of cardiovascular-related mortality in PRISm $(n=15)$. Another important finding was that the rate of $\mathrm{FEV}_{1}$ decline in incident PRISm was much higher than all other groups (including incident COPD). The study reports several interesting associations, for instance between incident heart failure and incident PRISm, that hold up after adjustment for multiple confounders.

The results are consistent with the previously reported analysis of the COPDGene study that showed PRISm is an unstable classification which is associated with increased mortality compared to GOLD 1 COPD $[12,13]$. At $12.4 \%-12.5 \%$, the prevalence of PRISm was higher in the COPDGene cohort than in the Rotterdam study, which might reflect different sampling schemes (clinical study centres in the former, population-based sampling in the latter) [12,13]. In a recent study in a large Danish cohort, ÇOLAK et al. [14] found that even when $\mathrm{FEV}_{1} / \mathrm{FVC}>0.8$, the presence of chronic respiratory symptoms is associated with respiratory-related hospitalisation and death. Remarkably, PRISm patients with such restrictive spirometric pattern seem to have a lower quality of life even when they are asymptomatic [15].

Some of the other conclusions of the study by WijnANT et al. [10] are the natural result of classifying a continuous process into discrete categories. For example, the authors concluded that persistent PRISm is associated with normal lung function decline. However, to be called persistent PRISm, individuals had to satisfy the definition of PRISm in both visits. Individuals who exited PRISm in the second visit (who either moved to control or to COPD groups), which constitute the majority of such individuals, were removed from this sub-analysis. This will selectively create a subgroup with relatively modest lung function decline.

The overall results suggest that in terms of outcomes such as lung function trajectories and mortality, PRISm course is closer to COPD than to those with normal spirometry. Given the high prevalence and the observed disease trajectory, the research and care provider communities can no longer ignore PRISm. General population longitudinal cohort studies such as the Rotterdam study provide a unique opportunity to shed light on the burden of COPD in subpopulations that are often excluded in randomised trials. Last year, the GOLD board of directors published a statement in the European Respiratory Journal asserting that "it is time for the world to take COPD seriously" [16]. We agree wholeheartedly with this statement, and believe that it is also timely for the research community to take more seriously the COPD subpopulations that are left out due to our (inevitably arbitrary) dichotomisation of continuous processes. The results presented by WIJNANT et al. [10] are the "prism" through which we can appreciate the broader "spectrum" of COPD.

Conflict of interest: A. Adibi has nothing to disclose. M. Sadatsafavi has nothing to disclose. 


\section{References}

1 GBD 2015 Chronic Respiratory Disease Collaborators. Global, regional, and national deaths, prevalence, disability-adjusted life years, and years lived with disability for chronic obstructive pulmonary disease and asthma, 1990-2015: a systematic analysis for the Global Burden of Disease Study 2015. Lancet Respir Med 2017; 5: 691-706.

2 Vogelmeier CF, Criner GJ, Martinez FJ, et al. Global Strategy for the Diagnosis, Management, and Prevention of Chronic Obstructive Lung Disease 2017 Report: GOLD Executive Summary. Am J Respir Crit Care Med 2017; 195: 557-582.

3 Mirza S, Benzo R. Chronic obstructive pulmonary disease phenotypes: implications for care. Mayo Clin Proc 2017; 92: 1104-1112.

4 Zafari Z, Sin DD, Postma DS, et al. Individualized prediction of lung-function decline in chronic obstructive pulmonary disease. CMAJ 2016; 188: 1004-1011.

5 Hurst JR, Vestbo J, Anzueto A, et al. Susceptibility to exacerbation in chronic obstructive pulmonary disease. $N$ Engl J Med 2010; 363: 1128-1138.

6 Johnson KM, Safari A, Tan WC, et al. Heterogeneity in the respiratory symptoms of patients with mild-to-moderate COPD. Int J Chron Obstruct Pulmon Dis 2018; 13: 3983-3995.

7 Han MK, Agusti A, Calverley PM, et al. Chronic obstructive pulmonary disease phenotypes. Am J Respir Crit Care Med 2010; 182: 598-604.

8 Burgel P-R, Paillasseur J-L, Janssens W, et al. A simple algorithm for the identification of clinical COPD phenotypes. Eur Respir J 2017; 50: 1701034.

9 Sin DD, Miravitlles M, Mannino DM, et al. What is asthma-COPD overlap syndrome? Towards a consensus definition from a round table discussion. Eur Respir J 2016; 48: 664-673.

10 Wijnant SRA, De Roos E, Kavousi M, et al. Trajectory and mortality of preserved ratio impaired spirometry: the Rotterdam Study. Eur Respir J 2020; 55: 1901217.

11 Ikram MA, Brusselle GGO, Murad SD, et al. The Rotterdam Study: 2018 update on objectives, design and main results. Eur J Epidemiol 2017; 32: 807-850.

12 Wan ES, Fortis S, Regan EA, et al. Longitudinal phenotypes and mortality in preserved ratio impaired spirometry in the COPDGene Study. Am I Respir Crit Care Med 2018; 198: 1397-1405.

13 Wan ES, Castaldi PJ, Cho MH, et al. Epidemiology, genetics, and subtyping of preserved ratio impaired spirometry (PRISm) in COPDGene. Respir Res 2014; 15: 89.

14 Çolak Y, Nordestgaard BG, Vestbo J, et al. Prognostic significance of chronic respiratory symptoms in individuals with normal spirometry. Eur Respir J 2019; 54: 1900734.

15 Guerra S, Carsin A-E, Keidel D, et al. Health-related quality of life and risk factors associated with spirometric restriction. Eur Respir J 2017; 49: 1602096.

16 Halpin DMG, Celli BR, Criner GJ, et al. It is time for the world to take COPD seriously: a statement from the GOLD board of directors. Eur Respir J 2019; 54: 1900914. 